\title{
Service Discovery from Observed Behavior while Guaranteeing Deadlock Freedom in Collaborations
}

\author{
Richard Müller ${ }^{1,2}$, Christian Stahl ${ }^{2}$, \\ Wil M.P. van der Aalst ${ }^{2,3}$, and Michael Westergaard ${ }^{2,3}$ \\ 1 Institut für Informatik, Humboldt-Universität zu Berlin, Germany \\ Richard.Mueller@informatik.hu-berlin.de \\ 2 Department of Mathematics and Computer Science, \\ Technische Universiteit Eindhoven, The Netherlands \\ $\{$ C.Stahl,W.M.P.v.d.Aalst, M. Westergaard\}@tue.nl \\ 3 National Research University Higher School of Economics, Moscow, 101000, Russia
}

\begin{abstract}
Process discovery techniques can be used to derive a process model from observed example behavior (i.e., an event $\log$ ). As the observed behavior is inherently incomplete and models may serve different purposes, four competing quality dimensions - fitness, precision, simplicity, and generalization - have to be balanced to produce a process model of high quality.

In this paper, we investigate the discovery of processes that are specified as services. Given a service $S$ and observed behavior of a service $P$ interacting with $S$, we discover a service model of $P$. Our algorithm balances the four quality dimensions based on user preferences. Moreover, unlike existing discovery approaches, we guarantees that the composition of $S$ and $P$ is deadlock free. The service discovery technique has been implemented in ProM and experiments using service models of industrial size demonstrate the scalability or our approach.
\end{abstract}

\section{Introduction}

Service-oriented design 24 reduces system complexity, and service models are useful to understand the running system, to verify the system's correctness, and to analyze its performance. However, it is often not realistic to assume that there exists a service model. Even if there exists a formal model of the implemented service, it can differ significantly from the actual implementation; The formal model may have been implemented incorrectly, or the implementation may have been changed over time. Fortunately, we can often observe behavior recorded in the form of an event log. Such event logs may be extracted from databases, message logs, or audit trails. Given an event log, there exist techniques to produce a (service) model. The term service discovery [5] or, more general, process discovery [3] has been coined for such techniques.

In this paper, we assume a known service model $S$ and an event $\log L$ containing observed behavior in the form of message sequences being exchanged between 
(instances of) the implementation of $S$ and (instances of) its environment (i.e., the services $S$ interacts with) to be given. Our goal is to produce a model of the environment of $S$. As the event $\log$ is inherently incomplete (i.e., not all possible behavior was necessarily observed), there are, in general, infinitely many models of the environment of $S$. Clearly, some models might be more appropriate than others regarding some user requirements. Therefore, service discovery can be seen as a search process: We search for a model of the environment that describes the observed behavior "best".

To judge the discovered model we consider two aspects: correctness (internal consistency of model, e.g., no deadlocks) and quality (ability to describe the underlying observed process well).

Correctness is motivated by the discovery of sound workflow models in [10, where soundness refers to the ability to always terminate [1]. In our serviceoriented setting, it is reasonable to require that $S$ and its environment interact correctly. As a minimal requirement of correct interaction, we assume deadlock freedom throughout this paper. We refer to such model of the environment of $S$ as a partner of $S$. Thus, we are interested in discovering a partner of $S$.

Regarding quality, there exist four quality dimensions for general process models [3]: (1) fitness (i.e., the discovered model should allow the behavior seen in the event log), (2) precision (i.e., the discovered model should not allow behavior completely unrelated to what was seen in the event $\log$ ), (3) generalization (i.e., the discovered model should generalize the example behavior seen in the event $\log$ ), and (4) simplicity (i.e., the discovered model should be as simple as possible). These quality dimensions compete with each other. For example, to improve the fitness of a model one may end up with a substantially more complex model. A more general model usually means a less precise model. We assume that a user guides the balancing of these four quality dimensions. As a consequence, we aim at discovering a service model that is a partner of $S$ and, in addition, balances the four quality dimensions guided by user preferences.

The actual challenge is now to find such a model. As a service $S$ has, in general, infinitely many partners, the search space for service discovery is infinite. Therefore, we are using a genetic algorithm to find a good but possibly not an optimal model of a partner of $S$. We have implemented this algorithm. It takes a service model $S$, an event $\log$, and values for the four quality dimensions as input. The output of the algorithm is a model of a partner of $S$ that comes close to the specified values of the quality dimensions. We show its applicability using eight service models of industrial size. Moreover, based on the notion of a finite representation of all partners of $S$ [14] - referred to as operating guideline-we additionally apply an abstraction that reduces the search space to a finite one. Although the abstraction only preserves fitness, our experimental results show that the other quality dimensions do not suffer too much due to this abstraction. An full version of this paper including a more detailed discussion on the abstraction and the experimental results has been published as a technical report in [22]. 
Summarizing, the main contributions of this paper are:

- adapting existing discovery techniques for workflows (i.e., closed systems) to services (i.e., reactive systems);

- adapting the metrics for the four quality dimensions to cope with service models;

- presenting an approach to reduce an infinite search space to a finite one; and

- validation of the algorithm based on a prototype.

We continue with a motivating example in Sect. 2, Section 3 provides background information on our formal service model and process discovery techniques. Section 4 adapts existing discovery techniques and metrics for workflows for service mining and reduces the infinite search space to a finite one. We present experimental results in Sect. 5. Section [6 reviews related work, and Section 7 concludes the paper.

\section{Motivating Example}

Figure 1 shows a service $S$ modeled as a state machine, and an event $\log L$. A transition label $! x(? x)$ denotes the sending (receiving) of a message $x$ to (from) the environment of $S$. The event $\log L$ contains information on 210 traces. There are three types of traces: $a c$ (10 times), ad (100 times), and $b d$ (100 times). Our goal is to produce a model of the environment of $S$. Two example models are $P$ and $R$ in Fig. 1. $P$ incorporates the frequently observed behavior in $L$ (traces $a d$ and $b d$ ) and disregards trace $a c$, arguing that $a c$ is negligible for a "good" model. $R$ incorporates even more than the observed behavior in $L$-for example, the trace $b c$ which was not observed in the interaction with $S$-generalizing the observed behavior in $L$ in account for $L$ 's incompleteness.

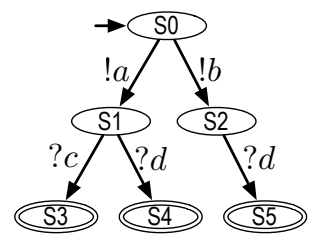

(a) Service $S$

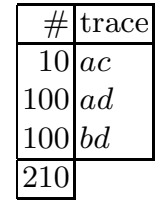

(b) Event $\log L$

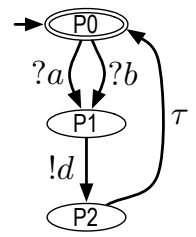

(c) Service $P$

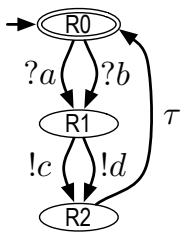

(d) Service $R$

Fig. 1. Running example: The event $\log L$ represents observed communication behavior of $S$ and its environment

The service $P$ is a partner of $S$ - they both interact without running into a deadlock - whereas the service $R$ is not: If $S$ sends a message $b$, then $R$ receives this message $b$ and may send a message $c$. However, $S$ cannot receive message $c$ and $R$ does not send any additional messages unless it receives a message $a$ or $b$. Thus, the interaction of $S$ and $R$ deadlocks. For this reason, we prefer $P$ over $R$ and our discovery algorithm would exclude $R$. Classical process mining approaches do not take $S$ into account and will allow for models that deadlock when composed. 


\section{Preliminaries}

For two sets $A$ and $B, A \uplus B$ denotes the disjoint union, i.e., writing $A \uplus B$ expresses the implicit assumption that $A$ and $B$ are disjoint. Let $\mathbb{N}^{+}$denote the positive integers. For a set $A,|A|$ denotes the cardinality of $A, \mathcal{B}(A)$ the set of all multisets (bags) over $A$, and [] the empty multiset. Throughout the paper, we assume a finite set of actions $\mathcal{A}$ such that $\{\tau$, final $\} \cap \mathcal{A}=\emptyset$.

For a set $A$, let $A^{*}$ be the set of finite sequences (words) over $A$. For two words $v$ and $w, v \sqsubseteq w$ denotes that $v$ is a prefix of $w$. For a ternary relation $R \subseteq A \times B \times A$, we shall use $a \stackrel{b}{\longrightarrow} R a^{\prime}$ to denote $\left(a, b, a^{\prime}\right) \in R$. If any of the elements $a, b$, or $a^{\prime}$ is omitted, we mean the existence of such an element. The relation $R^{*} \subseteq A \times B^{*} \times A$ is the reflexive and transitive closure of $R$, defined by $a \stackrel{b_{1} \ldots b_{n}}{\longrightarrow} R^{*} a^{\prime}$ if and only if there are $a_{0}, \ldots, a_{n} \in A$ such that $a=a_{0}, a^{\prime}=a_{n}$, and, for all $1 \leq i \leq n, a_{i-1} \stackrel{b_{i}}{\longrightarrow} a_{i}$. If $a \longrightarrow_{R^{*}} a^{\prime}$, then $a^{\prime}$ is reachable from $a$ in $R$.

\subsection{State Machines for Modeling Services}

We model a service as a state machine extended by an interface, thereby restricting ourselves to the service's communication protocol. An interface consists of two disjoint sets of input and output labels corresponding to asynchronous message channels. In the model, we abstract from data and identify each message by the label of its message channel.

Definition 1 (State Machine). A state machine $S=(Q, \alpha, \Omega, \delta, I, O)$ consists of a finite set $Q$ of states, an initial state $\alpha \in Q$, a set of final states $\Omega \subseteq Q$, a transition relation $\delta \subseteq Q \times(I \uplus O \uplus\{\tau\}) \times Q$, and two disjoint, finite sets of input labels $I \subseteq \mathcal{A}$ and output labels $O \subseteq \mathcal{A}$.

Let $l(t)=a$ define the label of a transition $t=\left(q, a, q^{\prime}\right) \in \delta$. We canonically extend $l$ to sequences of transitions. For a state $q \in Q$, define by en $(q)=\{a \mid$ $q \stackrel{a}{\longrightarrow} \delta\}$ the set of labels of outgoing transitions of $q$. The set $\mathcal{R}(S)=\left\{q \mid \alpha \longrightarrow \delta^{*}\right.$ $q\}$ denotes the reachable states of $S$. The state machine $S$ is deterministic if for all $q, q^{\prime}, q^{\prime \prime} \in Q$ and $a \in I \uplus O,\left(q, \tau, q^{\prime}\right) \in \delta$ implies $q=q^{\prime}$ and $\left(q, a, q^{\prime}\right),\left(q, a, q^{\prime \prime}\right) \in \delta$ implies $q^{\prime}=q^{\prime \prime}$; it is deadlock free if, for all $q \in \mathcal{R}(S)$, en $(q)=\emptyset$ implies $q \in \Omega$. $\lrcorner$

Graphically, we precede each transition label $x$ with ? (!) to denote an input (output) label. A final state is depicted with a double circle (e.g., $S_{3}$ in Fig. 1(a)). An incoming arc denotes the initial state (e.g., $S_{0}$ in Fig. 1(a)).

For the composition of state machines, we assume that their interfaces completely overlap. We refer to state machines that fulfill this property as composable. We compose two composable state machines $S$ and $R$ by building a product automaton $S \oplus R$, thereby turning all transitions into (internal) $\tau$-transitions. In addition, a multiset stores the pending messages between $S$ and $R$.

Definition 2 (Composition). Two state machines $S$ and $R$ are composable if $I_{S}=O_{R}$ and $O_{S}=I_{R}$. The composition of two composable state machines $S$ and 
$R$ is the state machine $S \oplus R=(Q, \alpha, \Omega, \delta, \emptyset, \emptyset)$ with $Q=Q_{S} \times Q_{R} \times \mathcal{B}\left(I_{S} \uplus I_{R}\right)$, $\alpha=\left(\alpha_{S}, \alpha_{R},[]\right), \Omega=\Omega_{S} \times \Omega_{R} \times\{[]\}, \delta$ containing exactly the following elements:

$$
\begin{aligned}
& \text { - }\left(q_{S}, q_{R}, B\right) \stackrel{\tau}{\longrightarrow} \delta\left(q_{S}^{\prime}, q_{R}, B\right) \text {, if } q_{S} \stackrel{\tau}{\longrightarrow} \delta_{S} q_{S}^{\prime}, \\
& \text { - }\left(q_{S}, q_{R}, B\right) \stackrel{\tau}{\longrightarrow} \delta\left(q_{S}, q_{R}^{\prime}, B\right) \text {, if } q_{R} \stackrel{\tau}{\longrightarrow} \delta_{R} q_{R}^{\prime} \text {, } \\
& \text { - }\left(q_{S}, q_{R}, B+[a]\right) \stackrel{\tau}{\longrightarrow} \delta\left(q_{S}^{\prime}, q_{R}, B\right) \text {, if } q_{S} \stackrel{a}{\longrightarrow} \delta_{S} q_{S}^{\prime} \text { and } a \in I_{S} \text {, } \\
& \text { - }\left(q_{S}, q_{R}, B+[a]\right) \stackrel{\tau}{\longrightarrow} \delta\left(q_{S}, q_{R}^{\prime}, B\right) \text {, if } q_{R} \stackrel{a}{\longrightarrow} \delta_{R} q_{R}^{\prime} \text { and } a \in I_{R} \text {, } \\
& \text { - }\left(q_{S}, q_{R}, B\right) \stackrel{\tau}{\longrightarrow} \delta\left(q_{S}^{\prime}, q_{R}, B+[a]\right) \text {, if } q_{S} \stackrel{a}{\longrightarrow} \delta_{S} q_{S}^{\prime} \text { and } a \in O_{S} \text {, and } \\
& \text { - }\left(q_{S}, q_{R}, B\right) \stackrel{\tau}{\longrightarrow} \delta\left(q_{S}, q_{R}^{\prime}, B+[a]\right) \text {, if } q_{R} \stackrel{a}{\longrightarrow} \delta_{R} q_{R}^{\prime} \text { and } a \in O_{R} \text {. }
\end{aligned}
$$

We compare two state machines $S$ and $R$ by a simulation relation, thereby treating $\tau$ like any action in $\mathcal{A}$. A binary relation $\varrho \subseteq Q_{S} \times Q_{R}$ is a simulation relation of $S$ by $R$ if (1) $\left(\alpha_{S}, \alpha_{R}\right) \in \varrho$, and (2) for all $\left(q_{S}, q_{R}\right) \in \varrho, a \in \mathcal{A} \uplus\{\tau\}$, $q_{S}^{\prime} \in Q_{S}$ such that $q_{S} \stackrel{a}{\longrightarrow} S q_{S}^{\prime}$, there exists a state $q_{R}^{\prime} \in Q_{R}$ such that $q_{R} \stackrel{a}{\longrightarrow} R$ $q_{R}^{\prime}$ and $\left(q_{S}^{\prime}, q_{R}^{\prime}\right) \in \varrho$. If such a $\varrho$ exists, we say that $R$ simulates $S$. A simulation relation $\varrho$ of $S$ by $R$ is minimal, if for all simulation relations $\varrho^{\prime}$ of $S$ by $R$, $\varrho \subseteq \varrho^{\prime}$.

We want the composition of two services to be correct. As a minimal criterion for correctness, we require deadlock freedom and that every reachable state contains only finitely many pending messages (i.e., the message channels are bounded). We refer to two services that interact correctly as partners.

Definition 3 (b-Partner). Let $b \in \mathbb{N}^{+}$. A state machine $R$ is a $b$-partner of a state machine $S$ if $S \oplus R$ is deadlock free and for all $\left(q_{S}, q_{R}, B\right) \in \mathcal{R}(S \oplus R)$ and all $a \in I_{S} \uplus I_{R}, B(a) \leq b$.

In Fig. 1 $P$ is a 1-partner of $S$, but $R$ is not because the composition $S \oplus R$ can deadlock.

If a state machine $S$ has one $b$-partner, then it has infinitely many $b$-partners. Lohmann et al. [14] introduce operating guidelines as a way to represent the infinite set of $b$-partners of $S$ in a finite manner. Technically, an operating guideline is a deterministic state machine $T$ where each state is annotated with a Boolean formula $\Phi$, which specifies the allowed combinations of outgoing transitions. A state machine $R$ is represented by an operating guideline if (1) there exists a minimal simulation relation $\varrho$ of $R$ by $T$ (as $T$ is deterministic, $\varrho$ is uniquely defined); and (2) for every pair of states $\left(q_{R}, q_{T}\right) \in \varrho$, the outgoing transitions of $q_{R}$ and the fact whether $q_{R}$ is a final state must define a satisfying assignment to $\Phi\left(q_{T}\right)$.

Definition 4 (b-Operating Guideline). An annotated state machine $(T, \Phi)$ consists of a deterministic state machine $T$ and a Boolean annotation $\Phi$, assigning to each state $q \in Q$ of $T$ a Boolean formula $\Phi(q)$ over the literals $I \uplus O \uplus\{\tau$, final $\}$.

A state machine $R$ matches with $(T, \Phi)$ if there exists a minimal simulation relation $\varrho$ of $R$ by $T$ such that for all $\left(q_{R}, q_{T}\right) \in \varrho, \Phi\left(q_{T}\right)$ evaluates to true for the following assignment $\beta$ : $\beta(a)=$ true if $a \neq$ final $\wedge q_{R} \stackrel{a}{\longrightarrow} \delta_{R}$ or $a=$ final $\wedge q_{R} \in$ $\Omega_{R}$, and $\beta(a)=$ false otherwise. 
Let $b \in \mathbb{N}^{+}$. The $b$-operating guideline $O G_{b}(S)$ of a state machine $S$ is an annotated state machine such that for all state machines $R, R$ matches with $O G_{b}(S)$ iff $R$ is a $b$-partner of $S$.

Figure 2a depicts $O G_{1}(S)=(T, \Phi)$ of the service $S$. The state machine $P$ (Fig. [1c) matches with $(T, \Phi)$ : The minimal simulation relation of $P$ by $T$ is $\varrho=$ $\left\{\left(P_{0}, T_{0}\right),\left(P_{1}, T_{3}\right),\left(P_{1}, T_{1}\right),\left(P_{2}, T_{5}\right),\left(P_{2}, T_{4}\right),\left(P_{0}, T_{5}\right),\left(P_{0}, T_{4}\right),\left(P_{1}, T_{7}\right),\left(P_{2}, T_{7}\right)\right.$, $\left.\left(P_{0}, T_{7}\right)\right\}$, and the formula $\Phi$ is evaluated to true, for all pairs of $\varrho$. For example, for $\left(P_{0}, T_{0}\right)$ we have $\Phi\left(P_{0}\right)=($ true $\vee$ false $) \wedge($ true $\vee$ false $)=$ true, and for $\left(P_{0}, T_{4}\right)$ we have $\Phi\left(T_{4}\right)=$ true. Thus, $P$ is a 1-partner of $S$. Figure $2 \mathrm{~b}$ depicts the smallest subgraph $G$ of $O G_{1}(S)$ such that $P$ is still simulated by $G$, i.e., the subgraph used for the simulation relation above. In contrast to $P$, the state machine $R$ (Fig. 1d does not match with $(T, \Phi)$, because $\left(R_{1}, T_{1}\right)$ violates the simulation relation: We have $R_{1} \stackrel{! c}{\longrightarrow}$ but $T_{1} \stackrel{! c}{\longrightarrow}$. Thus, $R$ is not a 1-partner of $S$.

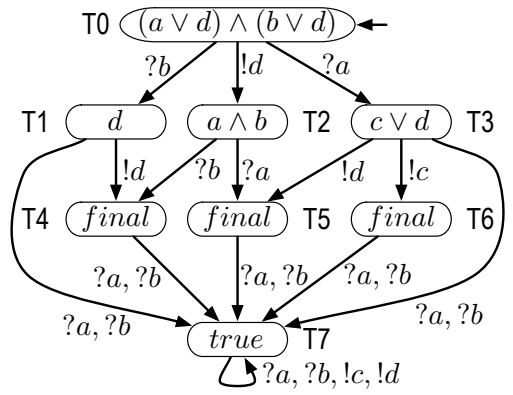

(a) $O G_{1}(S)$.

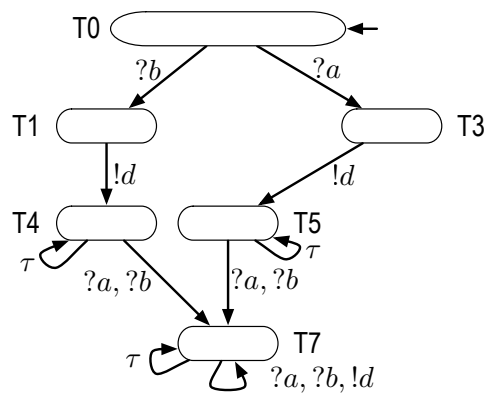

(b) Subgraph $G$ of $O G_{1}(S)$.

Fig. 2. $O G_{1}(S)$ and its smallest subgraph $G$ such that $P$ is simulated by $G$. The annotation of a state is depicted inside the state. For $O G_{1}(S)$, every state has a $\tau$ labeled self-loop and the annotation an additional disjunct $\tau$, which is omitted in the figure for reasons of readability.

In the remainder of the paper, we abstract from the actual bound chosen and use the terms partner and operating guideline rather than $b$-partner and $b$-operating guideline.

\subsection{Event Logs and Alignments}

An event $\log$ is a multiset of traces. Each trace describes the communication between $S$ and $R$ in a particular case in terms of a sequence of events (i.e., sent and received messages). We describe an event as an action label and abstract from extra information, such as the message content or the timestamp of the message. Formally, a trace $w \in \mathcal{A}^{*}$ is a sequence of actions, and $L \in \mathcal{B}\left(\mathcal{A}^{*}\right)$ is an event $\log$. 
To compare a (discovered) service model $R$ with the given event $\log L$, we use the alignment-based approach described in [4. This approach relates each trace $w \in L$ to a sequence $\sigma$ of transitions of $R$ that can be executed from $R$ 's initial state by pairing events in $w$ to events of $\sigma$.

Formally, a move is a pair $(x, y) \in\left((\mathcal{A} \uplus\{\gg\}) \times\left(\delta_{R} \uplus\{\gg\}\right)\right) \backslash\{(\gg, \gg)\}$. We call $(x, y)$ a move in the model if $x=\gg \wedge y \neq \gg$, a move in the log if $x \neq \gg \wedge y=\gg$, a synchronous move if $x \neq \gg \wedge y \neq \gg$, and a silent move if $x=\gg \wedge y \neq \gg \wedge l(y)=\tau$.

An alignment of a trace $w \in L$ to $R$ is a sequence $\gamma=\left(x_{1}, y_{1}\right) \ldots\left(x_{k}, y_{k}\right)$ of moves such that the projection of $\left(x_{1} \ldots x_{k}\right)$ to $\mathcal{A}$ is $w$, the projection of $\left(y_{1} \ldots y_{k}\right)$ to $\delta_{R}$ is $\left(\alpha_{R}, a_{1}, q_{1}\right) \ldots\left(q_{j-1}, a_{j}, q_{j}\right)$, and transition label $l\left(y_{i}\right)$ and action $x_{i}$ coincide for every synchronous move $\left(x_{i}, y_{i}\right)$ of $\gamma$. Let $\operatorname{trace}(\gamma) \in \mathcal{A}^{*}$ denote the word $l\left(y_{1}\right) \ldots l\left(y_{k}\right)$ with all $\tau$-labels removed.

Some alignments for $L$ and $P$ in Fig. 1 are:

$$
\gamma_{1}=\left|\begin{array}{c|c|}
a & c \\
\hline a \\
\left(P_{0}, a, P_{1}\right)
\end{array}\right| \gg\left|\gamma_{2}=\right| \begin{array}{c|c}
a & d \\
\hline a & d \\
\left(P_{0}, a, P_{1}\right) & \left(P_{1}, d, P_{2}\right)
\end{array}\left|\gamma_{3}=\right| \begin{array}{c|c|}
b & d \\
\hline\left(P_{0}, b, P_{1}\right) & \left(P_{1}, d, P_{2}\right)
\end{array} \mid
$$

The top row of $\gamma_{1}$ corresponds to the trace $a c \in L$ and the bottom two rows correspond to the service $P$. There are two bottom rows because multiple transitions of $P$ may have the same label; the upper bottom row consists of transition labels, and the lower bottom row consists of transitions. We have $\alpha_{P} \stackrel{a}{\longrightarrow} \delta_{P}^{*}$ but $\alpha_{P} \stackrel{a c}{\longrightarrow} \delta_{P}^{*}$; that is, $a c$ deviates from $a$ by an additional $c$-labeled transition. We denote this move in the log by a " $\gg$ " in the upper bottom row.

The goal is to find a best alignment that has as many synchronous and silent moves as possible. The approach in 4 finds such an alignment by using a cost function on moves. Let $\gamma$ be an alignment of a trace $w$ to $R$. Formally, a cost function $\kappa$ assigns to each move $(x, y)$ of an alignment $\gamma$ a cost $\kappa((x, y))$ such that a synchronous or silent move has cost 0 , and all other types of moves have cost $>0$. The cost of $\gamma$ is $\kappa(\gamma)=\sum_{i=1}^{k} \kappa\left(\left(x_{i}, y_{i}\right)\right) ; \gamma$ is a best alignment if, for all alignments $\gamma^{\prime}$ of $w$ to $R, \kappa\left(\gamma^{\prime}\right) \geq \kappa(\gamma)$. We use the function $\lambda_{R}$ to denote, for each trace $w \in L$, a best alignment of $w$ to $R$.

Finally, we combine the best alignment of each trace of $L$ to $R$ into a weighted automaton $A A$. A state of $A A$ encodes a sequence of (labels of) transitions of $R$. We define the weight $\omega(w)$ of each state $w$ as the number of times a trace of $L$ was aligned to $w$. We shall use $A A$ for the computation of metrics for the two quality dimensions precision and generalization later on.

Definition 5 (Alignment Automaton). The alignment automaton $A A(L, R)$ $=\left(V, v_{0}, E, \omega\right)$ of $L$ and $R$ consists of a set of states $V=\mathcal{A}^{*}$, an initial state $v_{0}=\varepsilon(\varepsilon$ is the empty trace), a transition relation $E \subseteq V \times \mathcal{A} \times V$ with $v \stackrel{a}{\longrightarrow} E v a$ iff there exists $w \in L$ such that $v a \sqsubseteq \operatorname{trace}\left(\lambda_{R}(w)\right)$, and a weight function $\omega: V \rightarrow \mathbb{N}^{+}$such that $\omega(v)=\sum_{w \in L \wedge v \sqsubseteq \operatorname{trace}\left(\lambda_{R}(w)\right)} L(w)$ for all $\left.v \in V.\right\lrcorner$

Figure 3 depicts the alignment automaton $A A(L, P)$ of the event $\log L$ and the state machine $P$. Each trace in $L$ is either aligned to the transition sequence 
labeled with $a$, ad or $b d$ (ignoring $\tau$ 's), as a transition sequence labeled with $a c$ is not present in $P$. The weight of each state is depicted inside the state; for example, $\omega(a)=110$ means 110 traces of $L$ can be aligned to a transition sequence of $P$ whose prefix is $a$.

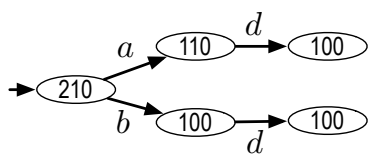

Fig. 3. The alignment automaton $A A(L, P)$

\section{Service Discovery from Observed Behavior}

Given a state machine $S$ and an event $\log L$, service discovery aims to produce a service $R$ that is (1) a partner of $S$ and (2) of high quality. The first requirement reduces the search space from all composable services to partners of $S$, and can be achieved by either model checking $S \oplus R$ or checking whether $R$ matches with the operating guideline $O G(S)$ of $S$. In the following, we discuss the second requirement.

\subsection{Incorporating the Quality Dimensions}

We are interested in discovering a partner of high quality. Numerous metrics for measuring the four quality dimensions have been developed [46]26. However, we cannot simply apply these metrics but have to adapt them to cope with service models.

Fitness. Let $R$ be a partner of $S$ and $L$ an event log. Fitness indicates how much of the behavior in the event $\log L$ is captured by the model $R$. A state machine with good fitness allows for most of the behavior seen in the event log. We redefine the cost-based fitness metrics from [4] for state machines: We quantify fitness as the total alignment costs for $L$ and $R$ (computed using the best alignments provided by $\lambda_{R}$ ) compared to the worst total alignment costs. The worst total alignment costs are just moves in the log for the events in the observed trace and no moves in the model, in all optimal alignments.

Definition 6 (Fitness). The fitness of $L$ and $R$ is defined by $f i t(L, R)=1-\frac{\sum_{w \in L}\left(L(w) \cdot \kappa\left(\lambda_{R}(w)\right)\right)}{\sum_{w \in L}\left(L(w) \cdot \sum_{x \in w} \kappa((x, \gg))\right)}$.

Assume a cost function $\kappa$ where each synchronous and each silent move has cost 0 , and all other types of moves have cost 1 . The best alignments given by $\lambda_{P}$ are $\gamma_{1}-\gamma_{3}$. We have costs of 1 for $\gamma_{1}, 0$ for $\gamma_{2}$, and 0 for $\gamma_{3}$; therefore, we calculate $f i t(L, P)=1-\frac{10 \cdot 1+100 \cdot 0+100 \cdot 0}{10 \cdot 2+100 \cdot 2+100 \cdot 2} \approx 0.976$. As expected, the fitness value is high because only 10 out of 210 traces are non-fitting traces in $L$ (i.e., the traces $a c)$. 
Simplicity. Simplicity refers to state machines minimal in structure, which clearly reflect the log's behavior. This dimension is related to Occam's Razor, which states that "one should not increase, beyond what is necessary, the number of entities required to explain anything." Various techniques exist to quantify model complexity [17. We define the complexity of the model $R$ by its number of states and transitions, and compare it with the smallest subgraph $G$ of $O G(S)$ such that $R$ is simulated by $G$. Although both $R$ and $G$ have the same behavior, $G$ is not necessarily less complex than $R$. Our metric takes this into account.

Definition 7 (Simplicity). Let $O G(S)=(T, \Phi)$. The simplicity $\operatorname{sim}(L, R)$ of $L$ and $R$ is $\frac{\left|Q_{G}\right|+\left|\delta_{G}\right|}{\left|Q_{R}\right|+\left|\delta_{R}\right|}$ if $\left|Q_{G}\right|+\left|\delta_{G}\right|<=\left|Q_{R}\right|+\left|\delta_{R}\right|$ and 1 otherwise, where $G$ is the smallest subgraph of $T$ such that $G$ simulates $R$.

Figure $2 \mathrm{~b}$ shows the smallest subgraph $G$ of $O G(S)$ such that $G$ simulates $P$. $G$ consists of 6 states and 14 transitions (including the $\tau$-loops at states $T_{4}, T_{5}$, and $T_{7}$ ). Therefore, $\left|Q_{G}\right|+\left|\delta_{G}\right|=6+14=20$ and $\left|Q_{P}\right|+\left|\delta_{P}\right|=3+4=7$; thus, $\operatorname{sim}(L, P)=1$. As expected, $L$ and $P$ have a perfect simplicity value, as $P$ is less complex than $G$.

Precision. Precision indicates whether a state machine is not too general. To avoid "underfitting", we prefer state machines with minimal behavior to represent the behavior observed in the event log as closely as possible. We redefine the alignment-based precision metric from [6] for state machines. This metric relies on building the alignment automaton $A A$, which relates executed and available actions after an aligned trace of the $\log$.

Definition 8 (Precision). Let $A A(L, R)=\left(V, v_{0}, E, \omega\right)$ be the alignment automaton of $L$ and $R$. Then the precision of $L$ and $R$ is defined by $\operatorname{pre}(L, R)=\left(\sum_{v \in V}(\omega(v) \cdot|\operatorname{exec}(v)|)\right) /\left(\sum_{v \in V}(\omega(v) \cdot|\operatorname{avail}(v)|)\right)$, where exec $(v)$ $=e n(v)$ in $A A(L, R)$, and $\operatorname{avail}(v)=\bigcup_{q \in X} e n(q)$ with $X=\left\{q \mid \alpha_{R} \stackrel{w}{\longrightarrow} \delta_{R}^{*}\right.$ $\left.q \wedge w_{\mid \mathcal{A}}=v\right\}$ in $R$.

Figure 3 shows the alignment automaton $A A(L, P)$, which has been build from the best alignments $\gamma_{1}-\gamma_{3}$. We obtain $\operatorname{pre}(L, P)=\frac{210 \cdot 2+110 \cdot 1+100 \cdot 0+100 \cdot 1+100 \cdot 0}{210 \cdot 2+110 \cdot 1+110 \cdot 2+100 \cdot 1+100 \cdot 2}=$ 0.6. As expected, $L$ and $P$ have average precision, as $P$ allows for far more behavior than the behavior observed in $L$.

Generalization. Generalization penalizes overly precise state machines which "overfit" the given log. In general, a state machine should not restrict behavior to just the behavior observed in the event log. Often only a fraction of the possible behavior has been observed, e.g., due to concurrency. For this dimension, we developed a new metric. We combine the generalization metric from [4] with the alignment automaton $A A(L, R)$. The idea is to use the estimated probability $\pi(x, y)$ that a next visit to a state $w$ of the alignment automaton will reveal a new trace not observed before: $x=|e n(w)|$ is the number of unique activities observed at leaving state $w$, and $y=\omega(w)$ is the number of times $w$ was visited by the event log. We employ an estimator for $\pi(x, y)$, which is inspired by 9 . 
Definition 9 (Generalization). Let $A A(L, R)=\left(V, v_{0}, E, \omega\right)$ be the alignment automaton of $L$ and $R$. The generalization of $L$ and $R$ is defined by $\operatorname{gen}(L, R)=1-\left(\frac{1}{|V|} \sum_{v \in V} \pi(|e n(v)|, \omega(v))\right)$, where $\pi$ can be approximated [4] by $\pi(x, y)=\frac{x(x+1)}{y(y-1)}$, if $y \geq x+2$, and $\pi(x, y)=1$, if $y \leq x+1$.

We obtain $\operatorname{gen}(L, P)=1-\frac{1}{5}\left(\frac{2 \cdot 3}{210 \cdot 209}+\frac{1 \cdot 2}{110 \cdot 109}+\frac{1 \cdot 2}{100 \cdot 99}\right) \approx 1$. Given the numbers of traces in $L, L$ and $P$ have nearly perfect generalization as expected, because it is unlikely to reveal a new trace not observed before.

Balancing the Quality Dimensions. As quality refers to the possibly competing quality dimensions fitness, simplicity, precision and generalization [3], we cannot assume the existence of a partner that has the highest value for every dimension. We rather need to balance these dimensions and, therefore, assume that a user specified his requirements using weights $\omega_{f i t}, \omega_{s i m}, \omega_{\text {pre }}$, and $\omega_{\text {gen }}$. With these four weights, we can actually search for the partner of $S$ that has highest quality.

Definition 10 (Quality). Let $\omega_{\text {all }}=\omega_{\text {fit }}+\omega_{\text {sim }}+\omega_{\text {pre }}+\omega_{\text {gen }}$. The quality of $R$ for $L$ is defined by quality $(L, R)=\frac{\omega_{\text {fit }}}{\omega_{\text {all }}} \operatorname{fit}(L, R)+\frac{\omega_{\text {sim }}}{\omega_{\text {all }}} \operatorname{sim}(L, R)+\frac{\omega_{\text {pre }}}{\omega_{\text {all }}} \operatorname{pre}(L, R)+$ $\frac{\omega_{g e n}}{\omega_{\text {all }}} \operatorname{gen}(L, R)$

Using weights of 2 for fitness, precision, and generalization, and a weight of 1 for simplicity (incorporating that the discovered service can be simpler than its simulation subgraph), we obtain quality $(L, P)=\frac{2}{7} \cdot 0.976+\frac{1}{7} \cdot 1+\frac{2}{7} \cdot 0.6+\frac{2}{7} \cdot 1 \approx$ 0.879 .

\subsection{A Finite Abstraction of the Search Space}

The actual challenge of service discovery is that the search space is the set partners of $S$, which is infinite. In the following, we present an abstraction that reduces the search space to a finite number of partners. To this end, we restrict ourselves to partners of $S$ that are valid subgraphs of $O G(S)=(T, \Phi)$, i.e., subgraphs of $T$ whose states are connected, contain the initial state of $T$, and that match with $O G(S)$. As $T$ contains only finitely many states, the number of valid subgraphs of $O G(S)$ is finite too. So, instead of investigating any partner of $S$, we only consider valid subgraphs of $O G(S)$.

However, this finite abstraction comes at a price: Although every valid subgraph is a partner of $S$, we may have excluded partners of $S$ that have a better quality than any valid subgraph. More precisely, it can be shown that this abstraction only preserves fitness. We do not elaborate on this and refer the interested reader to 22. The experimental results in Sect. 5 illustrate the appropriateness of the abstraction.

\subsection{Algorithm and Implementation}

Discovering a partner for a given state machine $S$ and an event $\log L$ is challenging because the search space is the infinite set of partners of $S$. Even the 
finite abstraction of the search space to valid subgraphs (see Sect.4.2) may still be too large to search for an optimal candidate exhaustively. Thus, we are using a genetic algorithm to find a partner of high but possibly not of highest quality. Genetic algorithms have been successfully applied for discovering workflow models [16 10]. A genetic algorithm evolves a population of candidate solutions (i.e., the individuals) step-wise (i.e., in generations) toward better solutions of an optimization problem. In our setting, an individual is a state machine $R$. The quality of a candidate solution is determined by the quality of $R$ (see Def. 10).

Our algorithm employs the general procedure of genetic algorithms, which is depicted in Fig. 4. It creates children through the operations crossover (i.e., randomly exchanging subgraphs between two given individuals), mutation (i.e., randomly adding or removing a transition or a final state from a given individual), and replacement (i.e., replacing a randomly chosen individual by a new, randomly generated individual). We employ a combination of four different termination criteria: A time and a generation limit (i.e., the evolution stops after a given amount of time or generations), a stagnation limit (i.e., the evolution stops if the quality of the high-quality individual stagnates a given number of generations), and a quality limit (i.e., the evolution stops if the high-quality individual meets a specified quality threshold).

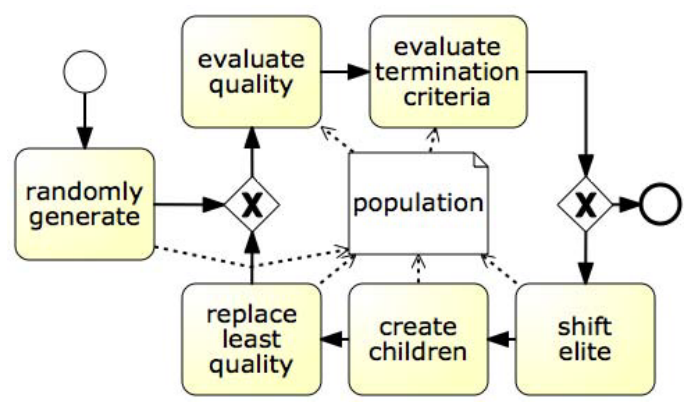

Fig. 4. The different phases of the genetic algorithm

We have implemented the genetic algorithm, with and without the abstraction presented in Sect. 4.2, as a runner-up package in the latest ProM 6.3 release1.

\section{Experimental Results}

In this section, we evaluate the feasibility of our approach by discovering partners for eight service models of industrial size. Table 1 gives an overview about the eight service models. The services "Loan Approval" and "Purchase Order" are taken from the WS-BPEL specification [12, all other examples are industrial service models provided by a consulting company.

${ }^{1}$ https://svn.win.tue.nl/trac/prom/wiki/rel63:release 
Table 1. Size of the service models, the operating guidelines, and event logs

\begin{tabular}{|c|c|c|c|c|c|}
\hline \multicolumn{3}{|l|}{ service $S$} & \multicolumn{2}{|c|}{$O G(S)$} & event $\log L$ \\
\hline name (abbreviation) & $|Q|$ & $|\delta|$ & $|Q|$ & $|\delta|$ & cases events \\
\hline Car Breakdown (CB) & 11,381 & 39,865 & , 449 & 13,863 & 3001,938 \\
\hline Deliver Goods (DG) & 148 & 13,832 & 377 & 13,838 & 3001,938 \\
\hline Loan Approval (LA) & 30 & 41 & 21 & 84 & 3002,537 \\
\hline Purchase Order (PO) & 402 & 955 & 169 & 1,182 & 3002,537 \\
\hline Internal Order (IO) & 1,516 & 4,996 & 97 & 567 & 3001,938 \\
\hline Ticket Reservation (TR) & 304 & 614 & 111 & 731 & 3002,381 \\
\hline Reservations (RS) & 28 & 33 & 370 & 3,083 & 3002,671 \\
\hline Contract Negotiation $(\mathrm{CN})$ & 784 & 1,959 & 577 & 4,859 & 3001,938 \\
\hline
\end{tabular}

As most services were specified in WS-BPEL, we had to translate them into state machines using the compiler BPEL2oWFN 13 . For each state machine $S$, we calculated the operating guideline $O G(S)$ using the tool Wendy [15]. Next, we used the underlying state machine $T$ of $O G(S)$ to generate a random event $\log L$ using the tool Locretia 2 . Because $T$ is the "most permissive" partner [14] of $S$, there exists a partner exhibiting the observed behavior in $L$. Each such event $\log L$ is free of noise and consists of 300 cases with about 1,900-2, 700 events. Table 1 shows the details. The size of our generated event logs is the size of event logs successfully applied to evaluate the genetic process discovery algorithm in [10. Finally, we used our implementation to discover a partner of $S$ from $O G(S)$ and $L$.

As parameters for the genetic algorithm, we used an initial population of 100 individuals, a mutation/crossover/replacement probability of 0.3 with at most 1 crossover point, and elitism of 0.3 , i.e., the 30 individuals with the highest quality are directly shifted to the next generation. The computation of a new generation stops after 1, 000 generations, if the highest quality stagnates for 750 generations, if a quality of 0.999 is reached, or if the algorithm ran for 60 minutes. To take into account that a discovered service can be simpler than the subgraph to be compared, we chose a weight of 1 for simplicity and a weight of 2 for all other dimensions. The experiment data is available online 3 .

To the best of our knowledge, there does not exist any other service discovery implementation with which we could compare our algorithm. Therefore, we performed two different experiments: discovering a partner from the complete search space (Experiment 1), and discovering a partner from the abstract search space (Experiment 2).

The results in Table 2 show that discovered partners in Experiment 1 are more complex than the ones in Experiment 2; that is, valid subgraphs are smaller than arbitrary partners. This explains the higher computation time in Experiment 1

\footnotetext{
2 http://svn.gna.org/viewcvs/service-tech/trunk/locretia/

3 https://u.hu-berlin.de/mueller
} 
by a factor of 1-44 compared to Experiment 2: Smaller candidates enable the algorithm to compute more generations in less time. For the same reason, Experiment 2 produced, in general, partners with higher fitness. The simplicity values are by Def. 6 higher for Experiment 2. In all examples, the discovered partners in Experiment 2 have slightly higher precision values than the partners discovered in Experiment 1. However, in three out of eight examples they have slightly lower generalization values. Restricting the search space to valid subgraphs is an abstraction, which neither preserves precision nor generalization. Therefore, we expected lower precision and generalization values for the partners discovered in Experiment 2. Despite the loss of preservation of the abstraction, the overall quality of the respective partner discovered in Experiment 2 is in all examples better.

Summing up, our experimental results validate that, in general, partner discovery produces better results on a finite abstraction of the search space than on the complete search space. Although the abstraction only preserves fitness, the values of the other three dimensions and the quality are high.

Table 2. Discovery of an ordinary partner (Experiment 1) and a valid subgraph (Experiment 2) using the genetic algorithm (with quality and time) conducted on a MacBook Pro, Intel Core i5 CPU with $2.4 \mathrm{GHz}$ and $8 \mathrm{~GB}$ of RAM.

\begin{tabular}{|c|c|c|c|c|c|c|c|c|c|c|c|c|c|}
\hline \multirow[b]{2}{*}{$S$} & \multicolumn{7}{|c|}{ discovered partner in Experiment 1} & \multicolumn{6}{|c|}{ discovered partner in Experiment 2} \\
\hline & $|Q|$ & $|\delta|$ & $q$ fit & $\operatorname{sim}$ & pre & gen & $\mathrm{t}$ in $s$ & $|Q| \quad|\delta|$ & $q$ fit & & pre & gen & $\mathrm{t}$ in $s$ \\
\hline & 548 & 10 & & & & & & 863 & & & & & \\
\hline & 246 & 829 & & & & & & 8231 & & & & & 1,763 \\
\hline & 15 & & & & & & & 1430 & & 1 & 0.9 & & 73 \\
\hline & 101 & & & 0.8 & 0. & 0.94 & & 33107 & & 1 & 1 & & 214 \\
\hline & 107 & & & & 0.9 & & & $9 \quad 11$ & 6 & 1 & 0.98 & & 3,021 \\
\hline TR & 29 & & & & 0.82 & 0.9 & 3,606 & 15 & & 1 & & & 143 \\
\hline $\mathrm{R}$ & 218 & 671 & & 0.98 & 0.8 & 1 & 3,6011 & 176582 & 71 & 1 & 0.91 & & 207 \\
\hline $\mathrm{CN}$ & 73 & 2200 & 620.7 & 1 & 0.61 & 0.35 & 3,798 & 742010 & .940 .86 & 1 & 0.97 & 0.94 & $=3,606$ \\
\hline
\end{tabular}

\section{Related Work}

The term "service discovery" describes techniques for finding a service model in a service repository in service-oriented architectures [24], and techniques for producing a service model from observed communication behavior of services [5]. In this paper, we refer to the latter. We investigated the discovery of a service model from observed communication behavior, which corresponds to a particular form of process mining [3]. Process mining research has been focused on workflows (i.e., closed systems) but during the last few years, process mining techniques have also been applied to services resulting in the term "service mining". Paper [2] reviews service mining research and identifies two main challenges regarding the discovery of services: (1) the correlation of instances of a service 
with instances of another service (e.g., 819]) and (2) the discovery of services based on observed behavior (e.g., [1125 23/727/8]). This paper contributes to the second challenge.

In 21, we considered with weak termination a stronger correctness criterion than deadlock freedom but solely focused on the fitness dimension, thus, ignored the three other quality dimensions. To make the discovery efficient, we do not discover a "best" model as in 21] but a model of high quality using a genetic algorithm. The idea of using an genetic algorithm is inspired by the work of Buijs et al. [10] on discovering sound workflow models while balancing the four conflicting quality dimensions. In Sect. 4, we discussed the relation of our metrics for these four quality dimensions and the metrics used in [10. For the simplicity metric, we used the structure of the operating guideline, which does not exist for workflow models. Correctness in our setting is deadlock freedom of the service composition, a weaker criterion than soundness in [10]. To deal with correctness in the setting of services, we assume a service $S$ to be given and we discover a partner of $S$ from observed behavior of $S$.

Musaraj et al. 23. correlate messages from an event log without correlation information and use this information in their discovery algorithm. In contrast, we abstract from correlation information and assume cases to be independent. Furthermore, our approach produces a partner of a given service model $S$ and balances the four conflicting quality dimensions guided by user preferences. Motahari-Nezhad et al. 20,18, only consider fitness (referenced as "recall"), simplicity and precision, and ignore generalization of the discovered service. Like Musaraj et al. 23], they do not assume a service model to be given and, thus, they cannot guarantee that their produced service model can interact correctly with its environment. Other approaches discover workflow models from service interaction [1] from interaction patterns [7 27. Whereas our algorithm produces a complete service model, [11/7/27] can only discover parts of a service.

\section{Conclusion and Future Work}

We presented a technique to discover a service model from a given service $S$ and observed behavior of a service $P$ interacting with $S$. Our technique produces a service model for $P$ that can interact correctly (no deadlocks) with $S$ and, in addition, balances the four conflicting quality dimensions (i.e., fitness, simplicity, precision, and generalization). As an additional improvement, we proposed an abstraction technique to reduce the infinite search space to a finite one. As an exhaustive search to find an optimal solution may still be intractable, we implemented our technique as a genetic algorithm. In a prototypical implementation, we experimented with several service models of industrial size. Our results showed that the algorithm finds (nearly) optimal solutions in acceptable time. It is worth mentioning that our approach is not restricted to service models but can discover arbitrary reactive systems.

In future work, we aim to extend our presented approach by improving the simplicity metrics, studying the impact of different weights of the quality dimensions on the quality of the discovered partner, and investigating how the 
abstraction technique based on valid subgraphs can be improved such that it preserves all metrics. We also plan to extend our approach to stronger correctness criteria than deadlock freedom, e.g., weak termination (i.e., the possibility to always terminate in a service composition).

Acknowledgement. Support from the Basic Research Program of the National Research University Higher School of Economics is gratefully acknowledged.

\section{References}

1. van der Aalst, W.M.P.: The application of Petri nets to workflow management. Journal of Circuits, Systems, and Computers 8(1), 21-66 (1998)

2. van der Aalst, W.M.P.: Service mining: Using process mining to discover, check, and improve service behavior. IEEE Transactions on Services Computing (2012)

3. van der Aalst, W.M.P.: Process Mining: Discovery, Conformance and Enhancement of Business Processes. Springer (2011)

4. van der Aalst, W.M.P., Adriansyah, A., van Dongen, B.F.: Replaying history on process models for conformance checking and performance analysis. Wiley Interdisciplinary Reviews: Data Mining and Knowledge Discovery 2(2), 182-192 (2012)

5. van der Aalst, W., et al.: Process mining manifesto. In: Daniel, F., Barkaoui, K., Dustdar, S. (eds.) BPM Workshops 2011, Part I. LNBIP, vol. 99, pp. 169-194. Springer, Heidelberg (2012)

6. Adriansyah, A., Munoz-Gama, J., Carmona, J., van Dongen, B.F., van der Aalst, W.M.P.: Alignment based precision checking. In: La Rosa, M., Soffer, P. (eds.) BPM Workshops 2012. LNBIP, vol. 132, pp. 137-149. Springer, Heidelberg (2013)

7. Asbagh, M., Abolhassani, H.: Web service usage mining: mining for executable sequences. In: WSEAS 2007, vol. 7, pp. 266-271 (2007)

8. Basu, S., Casati, F., Daniel, F.: Toward web service dependency discovery for SOA management. In: SCC 2008, vol. 2, pp. 422-429 (2008)

9. Boender, C., Rinnooy Kan, A.: A bayesian analysis of the number of cells of a multinomial distribution. The Statistician, 240-248 (1983)

10. Buijs, J.C.A.M., van Dongen, B.F., van der Aalst, W.M.P.: On the role of fitness, precision, generalization and simplicity in process discovery. In: Meersman, R., et al. (eds.) OTM 2012, Part I. LNCS, vol. 7565, pp. 305-322. Springer, Heidelberg (2012)

11. Dustdar, S., Gombotz, R.: Discovering web service workflows using web services interaction mining. Int. Journal of Business Process Integration and Management 1(4), 256-266 (2006)

12. Jordan, D., et al.: Web services business process execution language version 2.0. OASIS Standard 11 (2007)

13. Lohmann, N.: A feature-complete Petri net semantics for WS-BPEL 2.0. In: Dumas, M., Heckel, R. (eds.) WS-FM 2007. LNCS, vol. 4937, pp. 77-91. Springer, Heidelberg (2008)

14. Lohmann, N., Massuthe, P., Wolf, K.: Operating guidelines for finite-state services. In: Kleijn, J., Yakovlev, A. (eds.) ICATPN 2007. LNCS, vol. 4546, pp. 321-341. Springer, Heidelberg (2007)

15. Lohmann, N., Weinberg, D.: Wendy: A tool to synthesize partners for services. Fundam. Inform. 113(3-4), 295-311 (2011) 
16. Medeiros, A., Weijters, A., van der Aalst, W.M.P.: Genetic process mining: an experimental evaluation. Data Mining and Knowledge Discovery 14, 245-304 (2007)

17. Mendling, J., Neumann, G., van der Aalst, W.M.P.: Understanding the occurrence of errors in process models based on metrics. In: Meersman, R., Tari, Z. (eds.) OTM 2007, Part I. LNCS, vol. 4803, pp. 113-130. Springer, Heidelberg (2007)

18. Motahari-Nezhad, H.R., Saint-Paul, R., Benatallah, B.: Deriving protocol models from imperfect service conversation logs. IEEE Trans. Knowl. Data Eng. 20(12), 1683-1698 (2008)

19. Motahari Nezhad, H.R., Saint-Paul, R., Casati, F., Benatallah, B.: Event correlation for process discovery from web service interaction logs. The VLDB Journal 20(3), 417-444 (2010)

20. Motahari-Nezhad, H., Saint-Paul, R., Benatallah, B., Casati, F.: Protocol discovery from imperfect service interaction logs. In: 2013 IEEE 29th International Conference on Data Engineering (ICDE), pp. 1405-1409 (2007)

21. Müller, R., van der Aalst, W.M.P., Stahl, C.: Conformance checking of services using the best matching private view. In: ter Beek, M.H., Lohmann, N. (eds.) WS-FM 2012. LNCS, vol. 7843, pp. 49-68. Springer, Heidelberg (2013)

22. Müller, R., Stahl, C., van der Aalst, W.M.P., Westergaard, M.: Service discovery from observed behavior while guaranteeing deadlock freedom in collaborations. BPM Center Report BPM-13-12, BPMcenter.org (2013), http://bpmcenter.org/wp-content/uploads/reports/2013/BPM-13-12.pdf

23. Musaraj, K., Yoshida, T., Daniel, F., Hacid, M.S., Casati, F., Benatallah, B.: Message correlation and web service protocol mining from inaccurate logs. In: ICWS 2010, pp. 259-266 (2010)

24. Papazoglou, M.: Web Services - Principles and Technology. Prentice Hall (2008)

25. Rouached, M., Gaaloul, W., van der Aalst, W.M.P., Bhiri, S., Godart, C.: Web service mining and verification of properties: An approach based on event calculus. In: Meersman, R., Tari, Z. (eds.) OTM 2006. LNCS, vol. 4275, pp. 408-425. Springer, Heidelberg (2006)

26. Rozinat, A., van der Aalst, W.M.P.: Conformance checking of processes based on monitoring real behavior. Information Systems 33(1), 64-95 (2008)

27. Tang, R., Zou, Y.: An approach for mining web service composition patterns from execution logs. In: WSE 2010, pp. 53-62 (2010) 